\title{
A SURVEY OF SUBPIXEL EDGE DETECTION METHODS FOR IMAGES OF HEAT-EMITTING METAL SPECIMENS
}

\author{
ANNA FABIJAŃSKA \\ Institute of Applied Computer Science \\ Łódź University of Technology, Stefanowskiego 18/22, 90-924 Łódź, Poland \\ e-mail: an_fab@kis.p.lodz.pl
}

\begin{abstract}
In this paper the problem of accurate edge detection in images of heat-emitting specimens of metals is discussed. The images are provided by the computerized system for high temperature measurements of surface properties of metals and alloys. Subpixel edge detection is applied in the system considered in order to improve the accuracy of surface tension determination. A reconstructive method for subpixel edge detection is introduced. The method uses a Gaussian function in order to reconstruct the gradient function in the neighborhood of a coarse edge and to determine its subpixel position. Results of applying the proposed method in the measurement system considered are presented and compared with those obtained using different methods for subpixel edge detection.
\end{abstract}

Keywords: subpixel accuracy, edge detection, surface property, approximation, Gaussian function.

\section{Introduction}

Nowadays computer vision systems are commonly used in a wide range of applications to model the real world (Ranky, 2003; Obinata and Dutta, 2007). They use digital images to recognize and describe various phenomena of interest. The images are provided by video, digital cameras, radars or specialized sensors and are processed using advanced image processing and analysis algorithms (Batchelor and Whelan, 2002; Steger et al., 2008; Gocławski et al., 2009). However, visual representation of information contained in an image is highly redundant. Therefore, after converting an image into its digital representation, a detailed image analysis is carried out. The analysis aims at separating information significant to the user or the process from the entire image information. Edge detection plays an important role in this step. In many applications the accuracy of edge detection is the most important criterion considered while designing the vision system. This is also true in the case of the computerized system for high temperature measurements of surface properties of metals and alloys which is discussed in this paper. Because in this system the geometrical parameters of a specimen are related to the surface tension, the precision of edge detection is crucial for the accuracy of the measurements (Sankowski et al., 2000; Fabijańska and Sankowski, 2009).
Various methods for edge detection have already been proposed. Most commonly, they are image derivative based approaches which are sensitive to noise and blur (Gonzalez and Woods, 2007; Senthilkumaran and Rajesh, 2009; Oskoei and Hu, 2010). As a result, they produce approximate, inaccurate and ambiguous results. Moreover, traditional edge detectors all belong to the pixel level and their accuracy is limited by a discrete structure of a pixel grid. Hence, their precision does not exceed one pixel. This accuracy does not meet the requirements of modern vision systems.

Modern vision systems more and more often require the precision of tenths or hundredths of a pixel. Therefore, the development of subpixel techniques for edge detection has become one of the hotspots of the current research in image processing. Subpixel methods should overcome limitations brought by a discrete structure of a pixel grid and determine edge position inside a pixel. Some work on developing methods for subpixel edge detection has already been done. However, the major methods are still to be developed.

In this paper the problem of edge detection at subpixel level in images of heat-emitting objects is discussed. A reconstructive method for subpixel edge detection is introduced. The method uses a Gaussian function in order to reconstruct the gradient profile in the neighborhood of a coarse edge and to determine subpixel edge location. 
The proposed method can be especially useful in the case of industrial applications of vision systems where sources emitting intense radiation have to be monitored (e.g., metallurgy, welding, glass making).

The paper is organized as follows. Firstly, in Section 2 a brief introduction into subpixel edge detection is given. Next, in Section 3 the method for surface tension measurement considered in this paper is introduced. This is followed in Section 4 by the description of factors making the traditional edge detectors insufficient for the measurement system considered. Section 5 introduces the proposed approach to subpixel edge detection. Results of applying the proposed method to the class of images considered are presented and compared with other approaches in Section 6. Finally, Section 7 concludes the paper.

\section{Background on subpixel edge detection}

The discrete structure of the digital raster limits the accuracy of image registration and processing. Firstly, due to the mixed pixel problem, the image shape may be changed or unambiguously registered during image acquisition (Scott, 2010). This the case of pixels which overlap two or more regions. In the output image these pixels obtain one intensity resulting from the intensities of neighboring regions. The problem can be addressed by increasing the image resolution, although it will never be eliminated. This is explained in Fig. 1. Specifically, Fig. 1(a) presents the original scene covered by a pixel grid and in Fig. 1(b) the output image of the scene is shown. Figure 1(c) shows the results of increasing the image resolution.

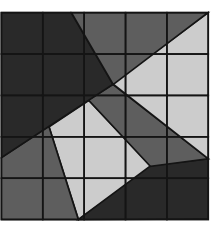

(a)

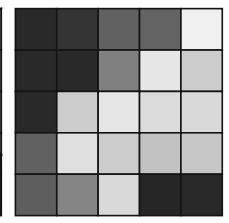

(b)

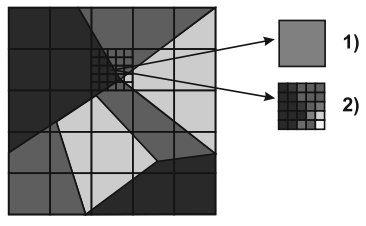

(c)
Fig. 1. Mixed pixel problem: original scene covered by a pixel grid (a), output image of the scene (b), results of increasing image resolution: 1 - pixel intensity for original resolution, 2-pixel intensities for quintuple the image resolution (c).

Because the traditional image processing algorithms regard pixel as a basic, indivisible image component, they are not able to divide a heterogeneous pixel into different classes but qualify it into one region. This decreases the accuracy of image processing and analysis.

The main idea behind subpixel edge detection is to overcome the above-mentioned limitations brought by a digital raster by performing soft classification in order to determine edge location inside a pixel and divide it into classes. However, it should be remembered that the discrete structure of a pixel grid significantly (and irreversibly) reduces edge information. Therefore, the subpixel edge position can only be approximated with some probability as its determination always requires guessing.

The need of subpixel accuracy in image processing and analysis was firstly pointed out in the late 1970s (Nevtia and Babu, 1978). Since then, the issue of edge detection at the subpixel level has gained attention of many scientists and researchers. Presently subpixel approaches to edge detection are still under development. However, the existing methods can be qualified into three main groups: curve-fitting methods, moment-based methods and reconstructive methods. They are briefly characterized in the following subsections.

2.1. Curve-fitting methods. Curve-fitting methods attempt to build a continuous border by fitting curves into edge points determined with pixel accuracy, using traditional edge detectors. The fitting is performed in an image plane (as sketched in Fig. 2).

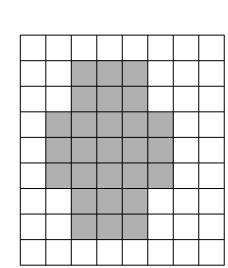

(a)

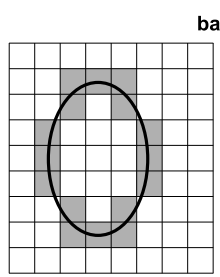

(b) adly defined edge point

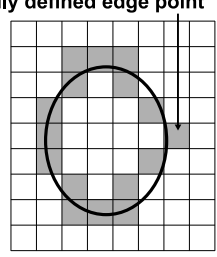

(c)

Fig. 2. Subpixel edge detection using curve fitting: input image (a), curve fitted into a properly detected edge (b), curve fitted into an edge with a badly defined edge pixel (c).

This methodology was used by Yao and Ju (2009), who fit cubic splines into spatial data points provided by the Canny operator, or by Breder et al.(2009), who used B-spline interpolation. A similar approach was also proposed by Kisworo et al. (1991) who applied deformable models for subpixel edge detection.

However, it should be underlined that the accuracy of curve-fitting methods strongly depends on the accuracy of border determination at the pixel level. This group of methods is also sensitive to badly defined edge points which can disturb the shape of the object (see Fig. 2(c)). Therefore, curve fitting methods can be successfully used only in applications where edges are well defined and the shape of the object is known a priori.

2.2. Moment-based methods. Moment-based methods use image moments to determine the location of edges. Intensity moments (based only on pixel intensities) and spatial moments (which use spatial information about the pixel neighborhood) can be differentiated. 
The first moment-based approach to subpixel edge detection was proposed by Machuca and Gilbert (1981). Their method integrates the region which contains the edge. Edge location is determined using moments found within the integrated region. The moments are defined based on properties of the vector from the given pixel to the center of gravity of the pixel square neighborhood.

Tabatabai and Mitchell (1984) proposed a method for subpixel edge detection which fits three intensity moments into the ideal step edge. The edge is defined as a sequence of intensity $h_{1}$ followed by a sequence of intensity $h_{2}$. The moments are defined as a sum of pixel intensity powers and do not consider any spatial information. The main drawback of this method is that it determines edges only in non-decreasing or non-increasing sequences of intensities.

The method developed by Lyvers et al. (1989) fits spatial (geometric) moments into a new 2D model of an ideal edge (see Fig. 3). In the model the edge is described by four parameters which determine its position with subpixel accuracy. These are the background intensity $h$, the intensity change at the edge $k$, the edge transition $l$ and the angle $\theta$ the edge makes with respect to $y$-axis.

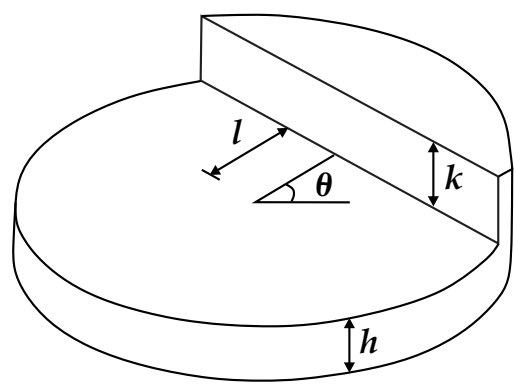

Fig. 3. Lyvers' edge model.

An ideal edge is then a step transition from intensity $h$ to intensity $h+k$ and is defined to lie within the unit circle at the distance of $l$ from the center of the edge model. In order to determine the subpixel edge position, pixels are mapped into this unit circle and a relationship between image moments and parameters of the edge is established.

The determination of edge parameters using Lyvers' approach requires evaluation of six moments using circular masks. This makes the method computationally complex. Moreover, moments used by the method are not orthogonal, which makes the method lack optimality in information redundancy. However, the edge model put into subpixel edge detection by Lavers was used by the succeeding approaches to the problem considered. Specifically, Ghosal and Mehrotra (1993) proposed a method which fits orthogonal Zernike moments into Lyvers' edge model. This decreased complexity of the method as only three masks were then required. However, Zernike moments have difficulties in describing small objects.
Recently, Bin et al. (2008) put forward Orthogonal Fourier-Mellin Moments (OFMMs) proposed by Sheng and Shen (1994) into subpixel edge detection. OFMMs are also fit into Lyvers' edge model. However, the method requires calculation of seven moments using circular masks, which makes it computationally complex.

The main drawback to moment-based approaches is the lack of clear criteria for classifying pixels as an edge or a non-edge. Moreover, they produce a response (i.e., parameters of subpixel edge) for every set of pixels containing a change in image intensity and work properly only in a close neighborhood of the edge pixel. If the edge position is more distant than the dimensions of the region of integration, moment based approaches fail. Therefore, in the current form they can only be used to refine the location of properly defined coarse edges.

\subsection{Reconstructive methods. Methods reconstruct-} ing the image intensity function determine subpixel edge location based on properties of the function which models the image intensity function at the edge. As an example, the approach by Xu (2009a) can be given. The method approximates image intensity at the edge using second order polynomial. Subpixel location of the edge is indicated by a point at the resulting curve where image intensity equals to the average of the intensities of the background and the object. However, methods reconstructing the image intensity function are in a minority.

Methods reconstructing the image first derivative attempt to build a continuous gradient function based on gradient sample values provided by operators like Sobel (Fabijańska and Sankowski, 2010), Prewitt (Bailey, 2005) or Canny (Rocket, 1999). The coordinates of the extremum of the reconstructed gradient function indicate the edge position with subpixel accuracy (see Fig. 4(e)). Most commonly, a second order polynomial is fit to gradient sample values in a small (3-5 pixels) neighborhood of a coarse edge (Bailey, 2005; Rocket, 1999; Fabijańska and Sankowski, 2010). Several approaches using the wavelet transform instead of the image first derivative have also been proposed (Liu et al., 2004; Xu, 2009b).

Methods reconstructing the image second derivative function retrieve a continuous function based on sample values provided by the LoG a operator. The zerocrossings of the reconstructed second derivative function determine edge locations with subpixel accuracy (see Fig. 4(f)). Most commonly, the image derivative function is linearly interpolated in the neighborhood where the second image derivative function changes its sign (Mac Vicar-Whelan and Binford, 1991; 1981). However, higher-order polynomials are also applied (Jin, 1990).

In order to reduce the computational complexity of edge detection methods, the image function is often reconstructed in some neighborhood of a coarse border. Therefore, a standard feature selection is first applied in order 
a)

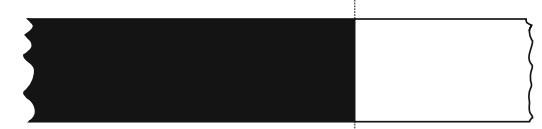

b)

c)
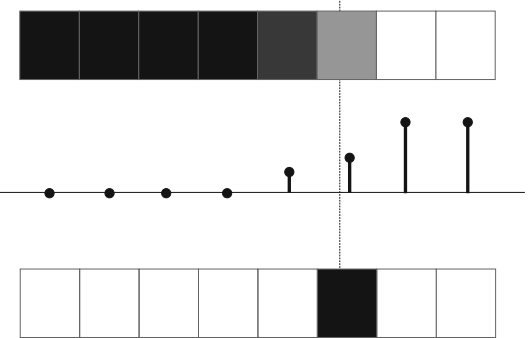

d)

e)

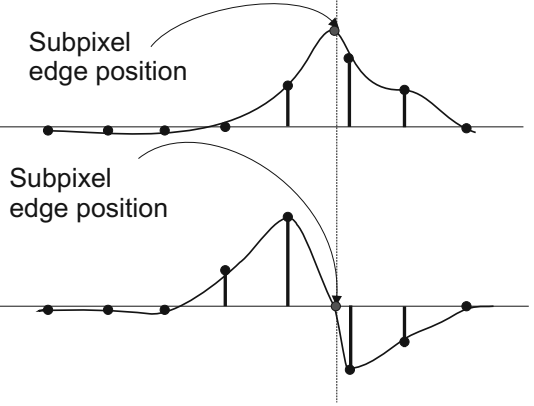

Fig. 4. Determination of the subpixel edge position using reconstructive approaches: real scene (a), image of the scene (b), intensity profile at the edge (c), coarse position of the edge (d), determination of the subpixel edge position using the first derivative (e), determination of subpixel edge position using the second derivative (f).

to determine a coarse location of the edge. Then this location is refined to the subpixel level adapting the local feature pattern in the closest neighborhood.

Among the discussed methods of subpixel edge detection, reconstructive approaches are most resistant to badly defined edge points. However, proper determination of the edge position is ensured only when the reconstructive function closely fits the image intensity profile at the edge.

2.4. Other methods. Several approaches to subpixel edge detection which do not meet the classification presented above have also been proposed. Stanke et al. (1998) proposed a method which determines the edge location at the subpixel level using the center of gravity of the gradient peak. A similar approach was also used by Ji et al. (2009). Kisworo et al. (1991), for determination of the subpixel edge position, used the image energy computed based on the image intensity and its Hilbert transform. An interesting approach was also introduced by Bie and Liu (2009). Their method decomposes pixels into subpixels using quad-tree decomposition.

Having in mind the classification presented above, the method introduced in this paper is a combination of reconstructive and curve fitting approaches. A more detailed description of the method is given in the following sections.

\section{Experimental set up}

The aim of the described research was to apply supbixel image processing in order to improve the accuracy of surface tension determination in the computerized system for high temperature measurements of surface properties of metals and alloys. In this section only a brief description of the measurement process is given. For more details regarding the system architecture and image processing algorithms applied during the measurements, the reader is referred to the works of Sankowski et al. (2000), Fabijańska and Sankowski (2009), Strzecha et al. (2010), as well as Koszmider et al. (2011).

The system considered determines surface tension of metals in a temperature up to $1800^{\circ} \mathrm{C}$. The measurements are based on images of heat-emitting, molten specimens of the investigated material. The images are registered with 256 levels of quantization and a spatial resolution of $240 \times 320$ pixels. Sample images obtained by the system are shown in Fig. 5. The left image (Fig. 5(a)) presents a specimen of copper at the temperature of $1180^{\circ} \mathrm{C}$. In the right image (Fig. 5(b)), a specimen of silver at temperature of $1042^{\circ} \mathrm{C}$ can be seen.

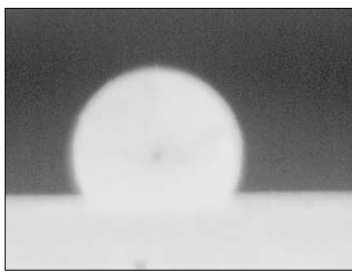

(a)

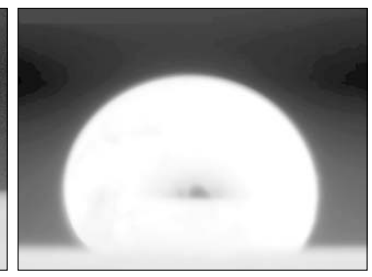

(b)
Fig. 5. Sample images of molten heat-emitting specimens: copper, $1180^{\circ} \mathrm{C}$ (a), silver, $1042^{\circ} \mathrm{C}$ (b).

In the measurement system considered the surface tension is determined using the sessile drop method (Huh and Reed, 1983; Hansen, 1993; Bachevsky et al., 1994; Adamson and Gast, 1997). The method relates characteristic dimensions of a drop of molten material and surface tension through (Extrand and Moon, 2010)

$$
h=\left[2\left(\frac{\gamma}{\rho g}\right)(1-\cos \theta)\right]^{\frac{1}{2}}\left[1+\left(\frac{\gamma}{\rho g}\right)^{\frac{1}{2}}\left(\frac{2}{a}\right)\right]^{-\frac{1}{2}}
$$

where $\gamma$ is the surface tension, $h$ is the drop height, $a$ is the base diameter, $\theta$ is the contact angle, $\rho$ is the density of liquid metal, $g$ is the acceleration gravity.

Drop characteristic dimensions are depicted in Fig. 6. 


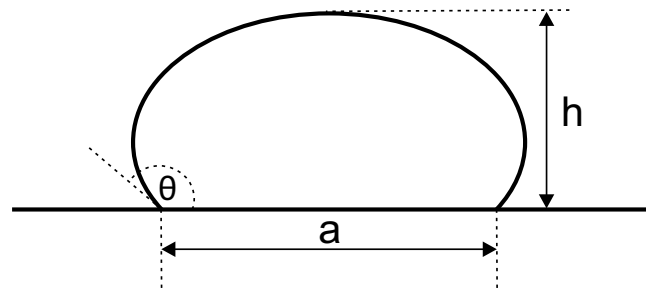

Fig. 6. Sessile drop and its geometrical parameters.

It can be easily seen that the more precisely the specimen geometrical parameters are determined, the more accurate the values of surface tension are obtained through Eqn. (1).

\section{Problem definition}

In the images considered, edges are surrounded by an aura i.e. glow which forms itself around the drop due to conditions of the measurements (high temperature, specimen intense thermal radiation, flow of protective gases, etc.). As a result, the border between the drop and the background becomes blurred and unsharp (see Fig. 7). This effect significantly hinders the proper determination of the drop edges and decreases the accuracy of drop shape description.

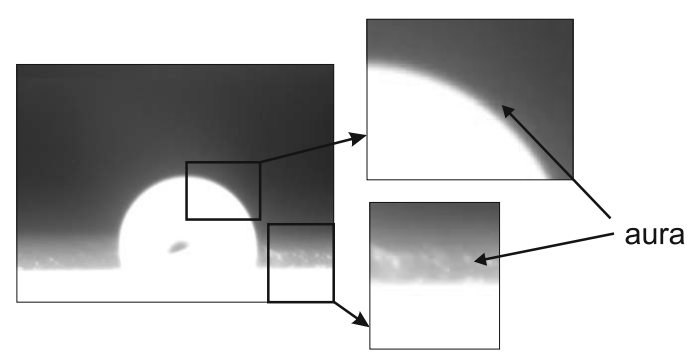

Fig. 7. Aura surrounding the specimen.

The boundary between the drop and the background is supposed to be located somewhere inside an aura. However, the classical approaches to edge detection fail to locate the border. Mostly, they either cut off the aura and decrease drop dimensions or join the aura with the object, which makes it increase. Additionally, traditional approaches to edge detection often falter when describing the specimen shape in contact of three phases (i.e., the solid phase of base, the liquid phase of metal and the gas phase of the environment). In all these cases, the accuracy of surface tension measurements is diminished. Moreover, different approaches to edge detection produce different results for one image. This problem is illustrated in Fig. 8 and Table 1. Figure 8 presents results of edge detection in the image of silver at $1042^{\circ} \mathrm{C}$ obtained using Sobel gradient masks (Fig. 8(a)), the Canny edge detector (Fig. 8(b)) and the LoG operator (Fig. 8(c)). Table 1 presents the cor-
Table 1. Influence of the edge position on the determined values of surface tension.

\begin{tabular}{|c|c|c|c|}
\hline Parameter [unit] & Sobel & Canny & LoG \\
\hline \hline$h$ [pixels] & 158 & 162 & 159 \\
\hline$a$ [pixels] & 196 & 190 & 188 \\
\hline$\theta\left[^{\circ}\right]$ & 126.5 & 125.7 & 127.6 \\
\hline$\gamma[\mathrm{mN} / \mathrm{m}]$ & 1014 & 934 & 921 \\
\hline
\end{tabular}

responding drop characteristic dimensions and values of surface tension.

The ambiguity of surface tension determination is unacceptable. Therefore, the main challenge of the proposed method was to modify the traditional edge detector in order to increase its aura resistance and the accuracy of surface tension determination. The proposed method on the one hand analyzes some neighborhood of the edge in order to reduce the influence of local intensity disturbances, and on the other brings the edge position to the subpixel level. A detailed description of the proposed method is given in the following section.

\section{Proposed approach}

5.1. Main idea. The proposed method attempts to reconstruct continuous edge information from the discrete image data. Specifically, the gradient profile at the coarse edge is reconstructed by fitting the Gaussian function into gradient sample values along the normal direction of the edge as shown in Fig. 9.

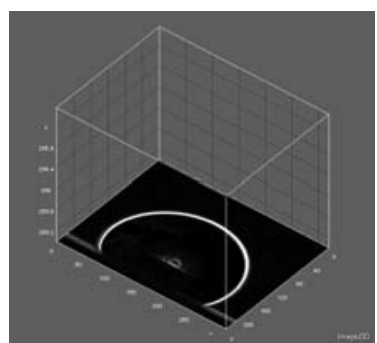

(a)

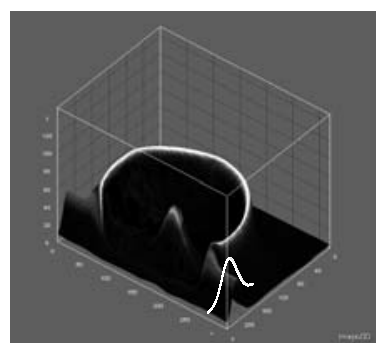

(b)
Fig. 9. Gradient profile reconstruction at the edge: top view on a gradient image (a), 3D surface plot of the gradient image with a Gaussian function (b).

Firstly, approximate discrete gradient sample values are obtained by convolving the input image $L$ with the horizontal $h_{x}$ and the vertical $h_{y}$ Sobel mask in accordance with

$$
\nabla L \approx \sqrt{\left(h_{x} \otimes L\right)^{2}+\left(h_{y} \otimes L\right)^{2}} .
$$

Next, the Gaussian function given by

$$
f(x)=A e^{\frac{-(x-\mu)^{2}}{2 \sigma_{G}^{2}}}
$$




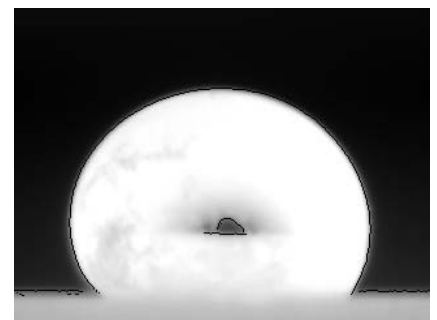

(a)

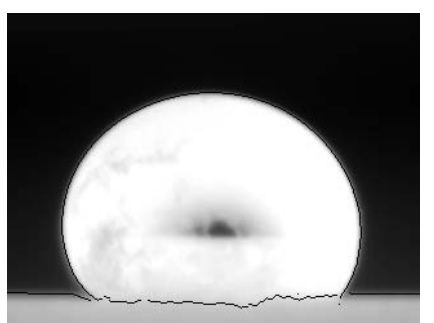

(b)

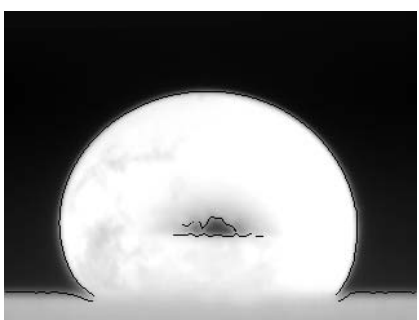

(c)

Fig. 8. Different results of edge detection obtained for one image: Sobel (a), Canny (b), LoG (c).

is fit to the gradient sample values along the normal direction of the coarse edge. Here $f(x)$ denotes the gradient value at the location $x$. Fitting aims at determining three parameters of the Gaussian function:

- $\sigma_{G}$, which describes the blur level of the edge;

- $A$, which corresponds to the maximum gradient value;

- $\mu$, which is the most important parameter as it indicates the subpixel location of the edge pixel.

The main idea of refining pixel location to the subpixel level using the Gaussian function is presented in Fig. 10. Discrete data points (empty circles) correspond to gradient sample values (shown under the graph) obtained for a pixel at location $x$. The parameter $\mu$ indicates the subpixel position of the pixel considered.

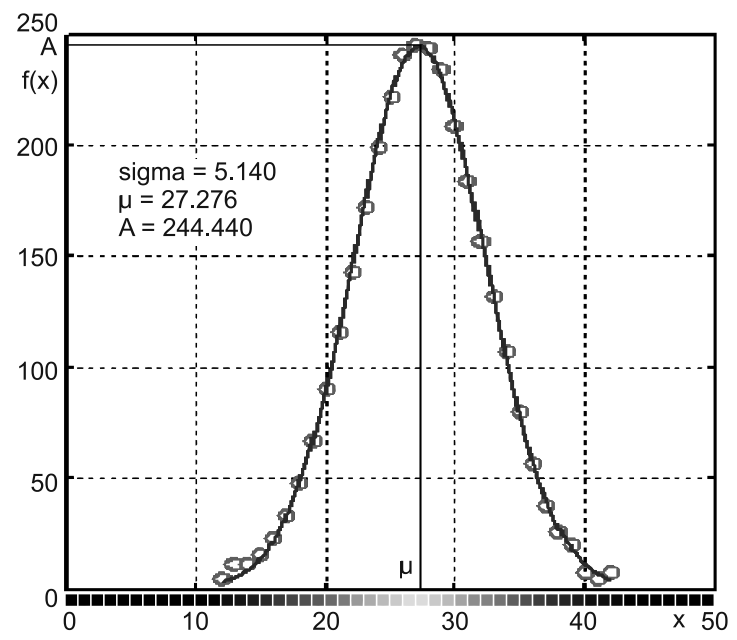

Fig. 10. Refining the pixel location to the subpixel level using a Gaussian function.

The fitting is performed in the nonlinear least squares sense. Specifically, the sum $S$ (see Eqn. (4)) of the squared differences between the observed gradient sample values $f_{i}$ (provided by Sobel masks) and the fit gradient values $f_{i}^{\prime}$ is minimized,

$$
S=\sum_{i=1}^{n}\left(f_{i}-f_{i}^{\prime}\right)^{2}
$$

where $n$ is the number of gradient sample values included in the fit. For optimization, the Gauss-Newton algorithm is used (Dennis and Schnabel, 1983). This method requires initialization. In order to ensure convergence, the initial values of the sought parameters should be close to the real ones. Therefore, in the given application, the following initialization is used:

$$
\left\{\begin{array}{l}
A=\max \left(f_{i}\right) \\
\mu=x \\
\sigma_{G}=n
\end{array}\right.
$$

where $x$ is the location of the coarse edge pixel being refined and $\max \left(f_{i}\right)$ is the maximum gradient sample value in the regarded neighborhood of $n$ pixels.

5.2. Drop shape description at the subpixel level. The proposed method was applied in a system for measurements of surface properties of metals and alloys in order to obtain the drop shape description at the subpixel level. Drop characteristic dimensions were obtained in the following steps:

- determination of the coarse edge;

- separation of the base line and the drop profile;

- determination of the subpixel position $b_{s}$ of the base line;

- determination of the drop profile at the subpixel level;

- determination of drop characteristic parameters.

They are described below. 


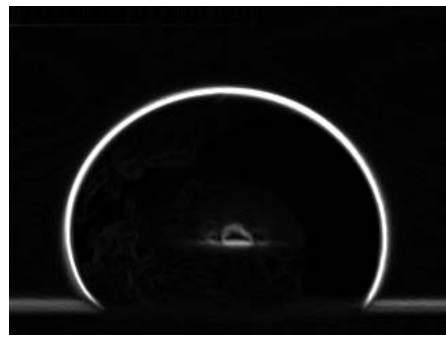

(a)

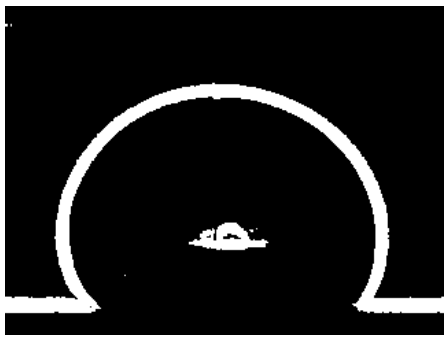

(b)

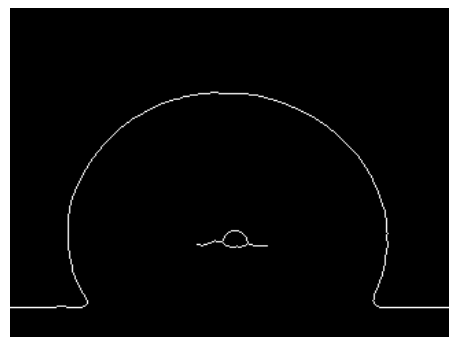

(c)

Fig. 11. Successive steps of coarse edge determination in sample image of silver from Fig 5(b): gradient image (a), gradient after thresholding (b), coarse edge—result of skeletonization (c).

Determination of the coarse edge. The coarse edge is determined using Sobel gradient masks. The input image $L$ is convolved with the horizontal $h_{x}$ and the vertical $h_{y}$ masks in accordance with Eqn. (2). The gradient image $\nabla L$ is then thresholded with a global threshold $F$ determined using the ISODATA algorithm (Ridler and Calvard, 1978). Thresholding performed in accordance with

$$
\nabla L^{\prime}(\mathbf{x})= \begin{cases}1 & \text { for } \quad \nabla L(\mathbf{x}) \geq F \\ 0 & \text { for } \quad \nabla L(\mathbf{x})<F\end{cases}
$$

produces a binary image corresponding with the highest gradient. Various thresholding procedures were tested (Otsu, C-means, Max-Entrophy, etc.). However, in the case of the regarded class of images, due to properties of the gradient image, the results of thresholding were similar. Therefore, finally the ISODATA method was used as a well established and fast approach which (in the case of the application considered) converges in several iterations.

In the next step the coarse edge $\delta L$ is obtained after skeletonisation performed on the binary image $\left|\nabla L^{\prime}\right|$ in accordance with (Sidiropoulos et al., 1992)

$$
\delta L=\bigcup_{n=0}^{N}\left[\left(\nabla L^{\prime} \ominus n H^{\prime}\right)-\left(\nabla L^{\prime} \ominus n H^{\prime}\right) \circ H\right]
$$

where $H$ denotes a convex structuring element, $\ominus$ and $\oplus$ denote erosion and dilation, respectively, o denotes morphological opening, and

$$
\begin{gathered}
N=\max \left\{n \mid \nabla L^{\prime} \ominus n H^{\prime} \neq \emptyset\right\}, \\
H^{\prime}=\{-h \mid h \in H\}, \\
n H=\left\{\begin{array}{cl}
\overbrace{\{\overline{0}\} \oplus H \oplus \cdots \oplus H}^{n} \text { for } n=1,2, \ldots, \\
\{\overline{0}\} & \text { for } n=0 .
\end{array}\right.
\end{gathered}
$$

In the case of the application considered, the skeletonization of a binarized gradient image always provides a continuous border between the background and specimen. No parameter setting is required. This automates the process of coarse edge extraction and eliminates additional processing like, for example, edge linking, which is required when hysteresis thresholding is used.

Successive steps of coarse edge determination in a sample image of silver (see Fig. 5(b)) are shown in Fig. 11. It can be seen (Fig. 5(c)), that the coarse edge is deformed in contact of three phases (i.e., the solid phase of base, the liquid phase of drop and the gas phase of atmosphere). The characteristic "roundoffs" of the specimen profile are caused by a strengthened aura phenomenon in this region.

When a skeleton is obtained, consecutive, connected pixels are linked together (from the bottom-left pixel to the top-right pixel) into a list e of points $e_{i}=\left(x_{e i}, y_{e i}\right)$ located along the coarse edge.

Separation of the base line and the drop profile. The list $\mathbf{e}$ of edge points obtained in the previous step is then divided into two sublists:

- list $\mathbf{b}$ of points $b_{i}=\left(x_{b i}, y_{b i}\right)$ belonging to the upper edge of the base plate;

- list $\mathbf{p}$ of points $p_{i}=\left(x_{p i}, y_{p i}\right)$ belonging to the drop profile.

The base plate is horizontal and the ordinate associated with the upper edge of the base plate in the image coordinate system is the most frequent one in the list e. Therefore, the list $\mathbf{b}$ of points belonging to the upper edge of the base plate is defined by the following equation:

$$
\mathbf{b}=\left\{e_{i}: y_{e i} \in\left[y_{b}-3, y_{b}+3\right] \& x_{e i} \notin\left[x_{\min }, x_{\max }\right]\right\},
$$

where

$$
y_{b}=\operatorname{mode}\left(y_{e i}\right)
$$

and $x_{\min }, x_{\max }$ denote abscissa of the leftmost and the rightmost point of the drop profile, respectively. The interval of three pixels is given in order to include all pixels in a close neighborhood of the base into calculations of the base level. Pixels located between $x_{\min }$ and $x_{\max }$ are excluded from calculations as their locations are distorted by an aura effect in contact of three phases. 
All points located above the level of a base plate are qualified to a specimen profile in accordance with

$$
\mathbf{p}=\left\{e_{i}: y_{e i}>y_{b}+3\right\}
$$

Again, an interval of three pixels is given to eliminate badly determined parts of the specimen profile in contact of three phases.

Points belonging to the coarse edge, the upper edge of a base plate and the drop profile obtained for exemplary image of silver from Fig. 5(b) are shown in Figure 12.

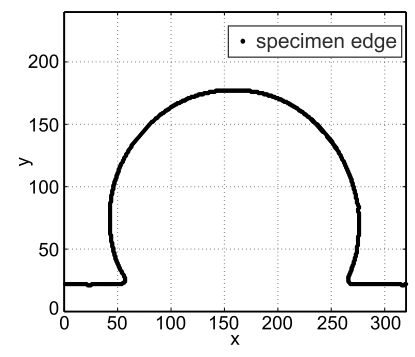

(a)

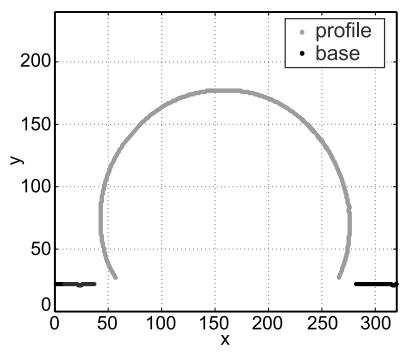

(b)
Fig. 12. Separation of the base line and drop profile from the coarse edge: coarse edge (a), base line and the drop profile after separation (b).

Determination of the subpixel position of the base line. Points qualified to the upper edge of the base plate are then used to determine the base subpixel location. Two steps need to be performed. First, the position of each point from list $\mathbf{b}=\left\{b_{i}\right\}$ is brought to the subpixel level by fitting a Gaussian function into a linear neighborhood of each point $b_{i}$ as described in Section 5.1. The fitting is performed in the vertical direction (see Fig. 13). Next, subpixel positions of points $b_{i}$ are approximated by a linear function $y=b_{s}$ determined by the least-squares fitting.

Determination of the drop profile at the subpixel level. Points qualified to the coarse profile are used to determine the profile subpixel location. Again, firstly the location of each point from list $\mathbf{p}=\left\{p_{i}\right\}$ is brought to the subpixel level by fitting a Gaussian function. The fitting is performed in the gradient direction. Specifically, in the case of points located in the upper part of the specimen profile, a vertical neighborhood is used. In the case of points located in the left and right parts of the specimen, the horizontal neighborhood is regarded (see Fig. 13).

In the next step, subpixel positions $p_{i}^{\prime}=\left(x_{p^{\prime} i}, y_{p^{\prime} i}\right)$, $i \in[0, k]$ of points $p_{i}$ are linked by a cubic spline to create a continuous curve. Specifically, spline interpolation is performed in accordance with

$$
P(x)=\sum P_{i}(x)
$$

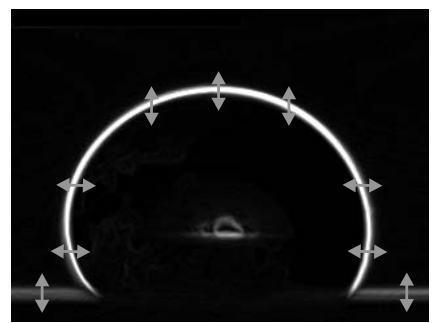

Fig. 13. Direction of gradient profile reconstruction in different parts of the specimen profile.

where $x \in\left[x_{i}, x_{i+1}\right], x_{p^{\prime} 0}=x_{0} \leq x_{1} \leq \cdots \leq x_{n-1} \leq$ $x_{n}=x_{p^{\prime} k}$ and

$$
\begin{aligned}
P_{i}(x)= & a_{i}\left(x-x_{i}\right)^{3}+b_{i}\left(x-x_{i}\right)^{2} \\
& +c_{i}\left(x-x_{i}\right)+d_{i}, \\
P_{i}\left(x_{i}\right)= & y_{i}, \quad P_{i}\left(x_{i+1}\right)=y_{i+1}, \\
P_{i-1}^{\prime}\left(x_{i}\right)= & P_{i}^{\prime}\left(x_{i}\right), \quad P_{i-1}^{\prime \prime}\left(x_{i}\right)=P_{i}^{\prime \prime}\left(x_{i}\right), \\
P_{0}^{\prime \prime}\left(x_{0}\right)= & 0, \quad P_{n-1}^{\prime \prime}\left(x_{n}\right)=0 .
\end{aligned}
$$

Intervals of five successive points are taken into account while determining consecutive splines.

Results of drop shape determination in a sample image from Fig. 5(b) are presented in Fig. 14. Specifically, Fig. 14(a) shows edges obtained at the pixel level compared with the original image. In Fig. 14(b), results of refining edges to the subpixel level are shown. Finally, Fig. 14(c) compares specimen edges obtained at the pixel (gray line) and subpixel (black line) level.

A qualitative assessment of the results presented in Fig. 14 shows clearly that the proposed method significantly improves the accuracy of specimen shape determination. The advantage of the proposed method is visible especially in contact of the specimen, the base plate and the background. While the coarse edge rounds the specimen profile in this region, the subpixel edge fits the specimen profile accurately. This is important for contact angle determination performed in the following step.

Determination of drop characteristic parameters. Finally, specimen characteristic dimensions were determined based on the subpixel shape description obtained in the previous steps. The specimen height $h$ is defined as the difference between the specimen maximum height and the subpixel base level $b_{s}$ as described by

$$
h=\max \left(y_{p^{\prime} i}\right)-b_{s}
$$

and shown in Fig. 15. The base diameter $a$ is the distance between the points $A_{0}$ and $A_{k}$ of the intersection of the linear function $y=b_{s}$ (approximating the upper edge of the base plate) with splines $P_{0}(x)$ and $P_{k}(x)$ (extrapolating the lowest parts of the left and right specimen profiles, respectively). 


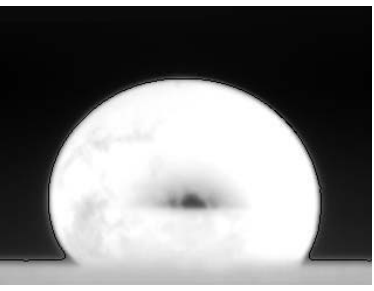

(a)

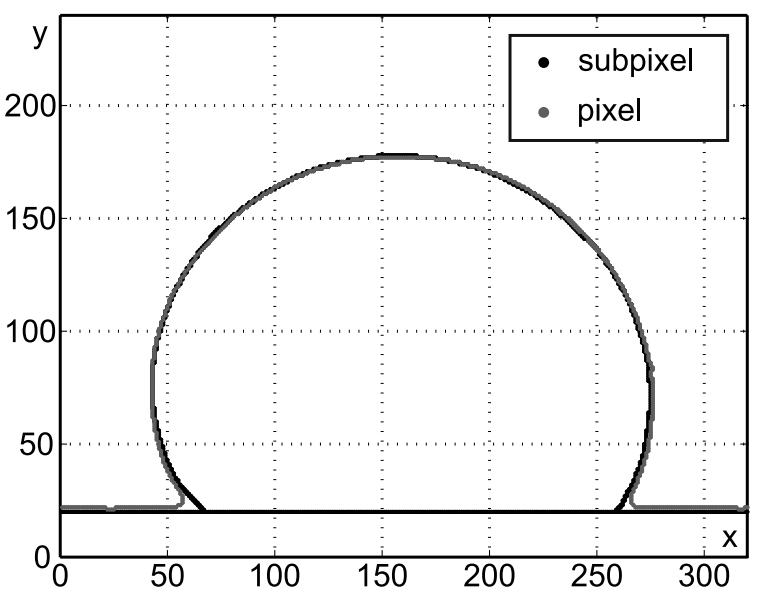

(c)

Fig. 14. Results of specimen shape determination at the subpixel level.

Finally, the contact angle $\theta$ is determined as the mean of angles $\theta_{1}$ and $\theta_{2}$ between the upper edge of the base plate and the tangents to the specimen profile in points $A_{0}$ and $A_{k}$. These are points of contact of three phases (i.e. the solid phase of base, the liquid phase of molten metal and the gas phase of atmosphere).

\section{Results and discussion}

6.1. Method performance analysis. In this section, results of edge detection in sample images of heatemitting objects provided by the proposed method are presented and compared with those provided by other ap-

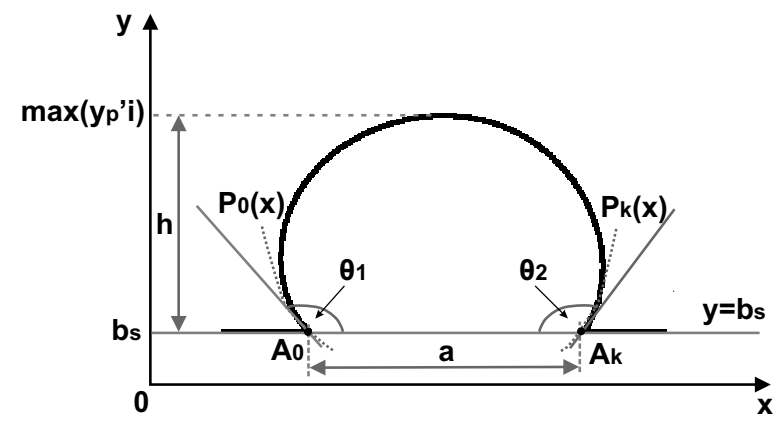

Fig. 15. Determination of specimen geometrical parameters. proaches. Specifically, the following methods (described in Section 2) are considered:

- geometrical moments approach;

- Zernike moments approach;

- orthogonal Fourier-Mellin moments approach;

- gravity center approach;

- gradient reconstruction using a parabola.

In all cases the procedure described in Section 5.2 was used to obtain the coarse edge. The above-mentioned methods were then used to refine the edge position to the subpixel level. Due to the limitations of the discrete structure of a pixel grid, the resulting subpixel edges are presented as plots in the Cartesian coordinate system. The bottom left corner of the image is located at the origin (point $(0,0)$ ) of the coordinate system while the top right corner corresponds to the point $(320,240)$.

First, moment based approaches were considered. Results of edge points detection at the subpixel level in a sample image from Fig. 5(b) are shown in Fig. 16.

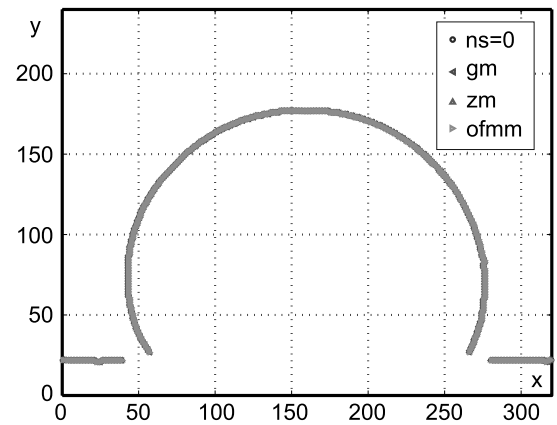

(a)

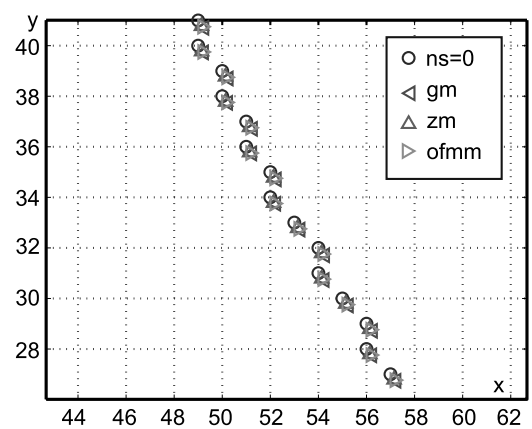

(b)

Fig. 16. Results of subpixel edge detection in the image from Fig. 5(b) using moment-based approaches: profile and base (a), magnified profile in contact of three phases (b).

Specifically, Fig. 16(a) presents the specimen profile and base while Fig. 16(b) shows magnified specimen profile in contact of three phases. The geometrical moment 
approach (series $g m$ ), the Zernike moment approach (series $z m$ ) and the orthogonal Fourier-Mellin moment approach (series ofmm) are analysed. Coarse locations of edge points corresponds to the series $n s=0$.

An analysis of the obtained results revealed that the moment based approaches fail when applied to the discussed class of images. All tested methods, i.e., geometrical moments, Zernike moments and orthogonal FourierMellin moments based approaches, produce very similar results and all subpixel positions are almost equally distant from the coarse edge. Additionally, this distance is close to zero so the edge location is almost unchanged. The position of badly determined edge points is not corrected. Due to these facts, moment based approaches were excluded from further analysis.

Results of edge detection at the subpixel level using the gravity center approach, the parabola fitting approach and the Gaussian function fitting approach (proposed in this paper) are presented in Figs. 17-19, respectively. The consecutive subfigures show the profile, the magnified profile in contact of three phases and the base, respectively. Again, the image of silver from Fig. 5(b) is used as an example. The obtained subpixel locations of the edge points are given as a function of neighborhood size considered during gradient profile reconstruction. Consecutive series $n s$ correspond to the number of gradient sample values used for determination of the subpixel position of an edge point. Specifically, $n s=w$ means that $w$ gradient sample values on each side of the coarse edge were used (the total number of sample values was $2 w+1)$. The series $n s=0$ corresponds to coarse edge points. The corresponding base positions $b_{s}$ are given in Table 2.

Table 2. Influence of the number of gradient sample values on the determined subpixel position of the base.

\begin{tabular}{|c|c|c|c|}
\hline & \multicolumn{3}{|c|}{ Method } \\
\hline$n s$ & GC & PAR & GAUSS \\
\hline \hline 0 & \multicolumn{3}{|c|}{22} \\
\hline 1 & 21.9499 & $-3.1 \mathrm{e}+011$ & 23.8159 \\
\hline 3 & 21.9839 & 22.2231 & 22.2093 \\
\hline 5 & 21.7877 & 21.7133 & 21.5778 \\
\hline 7 & 21.6409 & 21.6281 & 21.4647 \\
\hline 9 & 21.6508 & 21.7188 & 21.4647 \\
\hline 11 & 21.7088 & 21.7849 & 21.4647 \\
\hline
\end{tabular}

The results presented in Figs. 17-19 and Table 2 show that the determined subpixel edge position depends on the size of the neighborhood of the coarse edge used while refining the edge position. For small neighborhoods $(n s=1,3)$ reconstructive methods (i.e., parabola fitting and Gaussian function fitting) become unstable and in some cases produce a subpixel edge position significantly distant from the coarse edge. The instability appears in the cases when the local gradient peak is not at the location indicated by the coarse edge. The stability of both methods increases with an increase in the neighborhood size. However, while the subpixel edge position determined using the parabola fitting approach still changes with the neighborhood size, results provided by the Gaussian fitting approach become constant for appropriately big neighborhoods. In the case of the example presented in Fig. 19, the position of the edge converged for $n s=5$. Additionally, with an increase in the neighborhood size, the error of fitting the parabola into gradient sample values also increases. This is illustrated in Fig. 20. The parabola fits gradient sample values accurately only for small neighborhoods. This makes the parabola fitting approach inaccurate, unreliable and appropriate only for refining precisely determined coarse edges. The precision of Gaussian function fitting is high regardless of the neighborhood size, and hence the method is much more universal.

In the case of reconstructive approaches (i.e., parabola fitting and Gaussian function fitting) the usage of the smallest neighborhood ( $n s=1$ ) results in the highest method instability. The gravity center approach is most stable within the regarded methods as it always provides an edge located in the central part of the neighborhood considered. However, as in the case of the parabola fitting approach, the determined subpixel edge location changes for different neighborhoods, which makes the method less reliable than the Gaussian function fitting approach.

Despite the general properties discussed above, in the case of images of heat-emitting objects the proposed approach based on Gaussian fitting to a gradient function at the edge is much more robust in edge detection than the gravity center approach and the parabola fitting approach. The advantage of the Gaussian fitting approach is particularly evident in contact of three phases (which is particularly important in the discussed application).

In Fig. 21, results of a complete edge detection procedure in the images from Fig. 5 are shown. Edges obtained using the gravity center approach (for $n s=11$ ), the parabola fitting approach (for $n s=3$ ) and the proposed method (for $n s=11$ ) are presented and compared with the original images. In the case of reconstructive methods (i.e., parabola fitting and Gaussian function fitting), the size of the neighborhood was chosen in a way providing the closest and the most reliable fitting of a function into the gradient profile.

The bottom panel corresponds to the image of copper from Fig. 5(a). The top panel corresponds to the image of silver from Fig. 5(b). In the first column (Fig. 21(a)) results obtained using the gravity center approach are shown. The second column (Fig. 21(b)) corresponds to results provided by the Gaussian function fitting approach. Finally, in the third column (Fig. 21(c)), results of the parabola fitting approach are presented. Due to the limitations of a pixel grid, in all cases the edge position was 


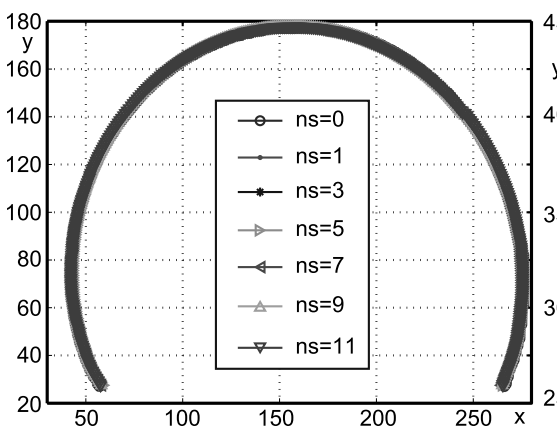

(a)

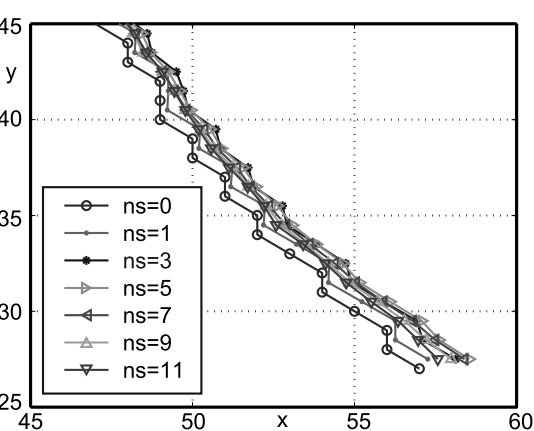

(b)

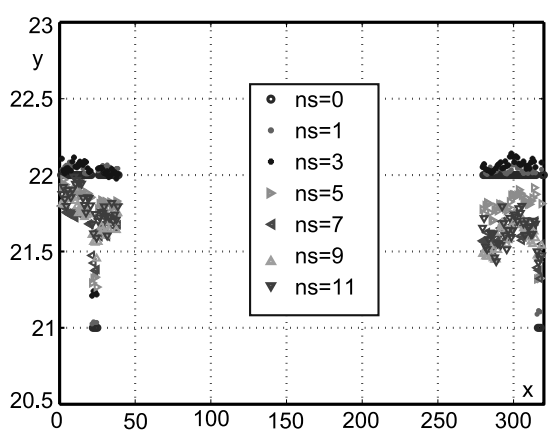

(c)

Fig. 17. Results of subpixel edge detection in the image from Fig. 5(b) using the gravity center approach: profile (a), contact of three phases (b), base (c).

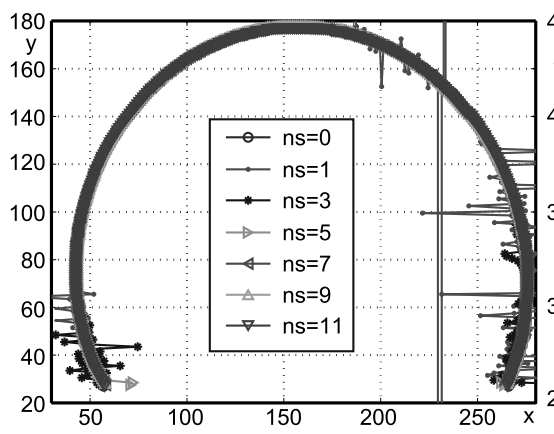

(a)

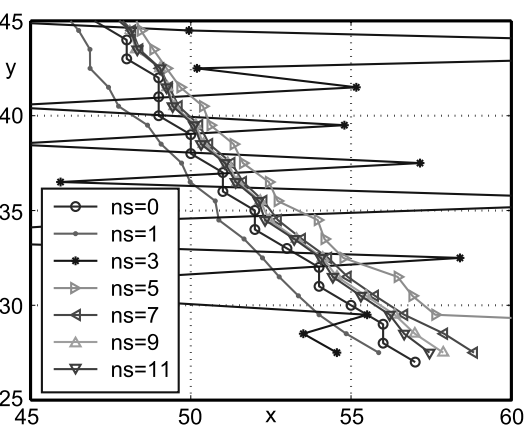

(b)

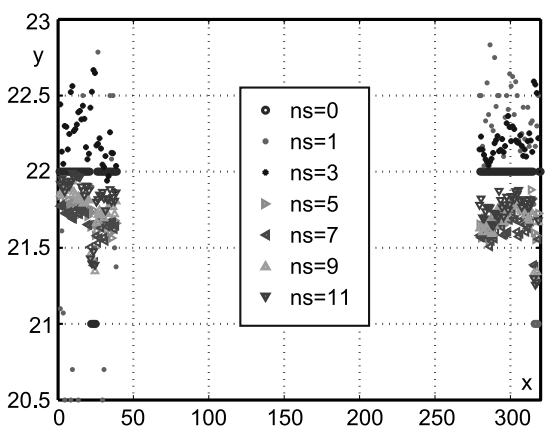

(c)

Fig. 18. Results of subpixel edge detection in the image from Fig. 5(b) using the parabola fitting approach: profile (a), contact of three phases (b), base (c).

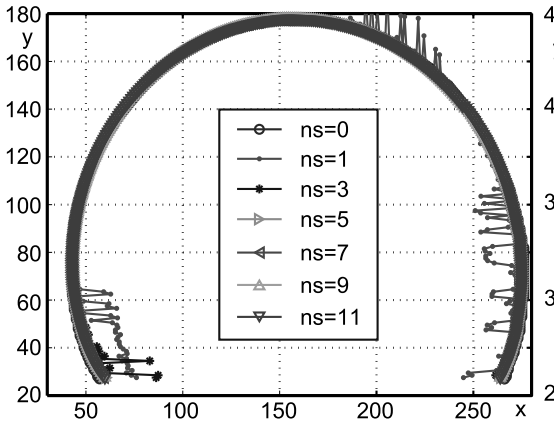

(a)

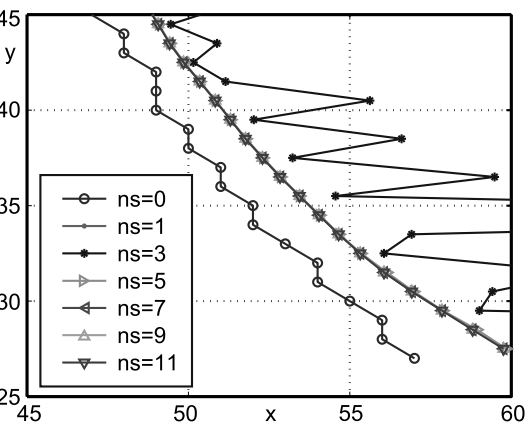

(b)

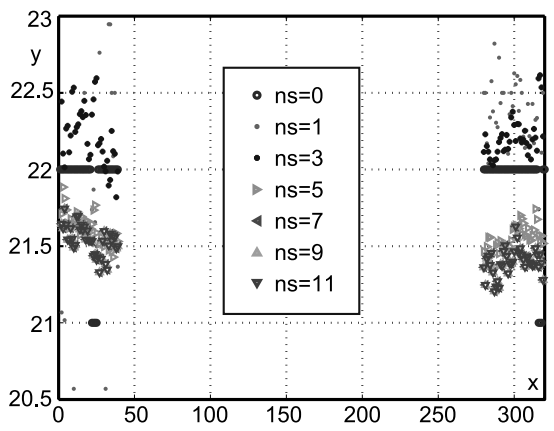

(c)

Fig. 19. Results of subpixel edge detection in the image from Fig. 5(b) using the Gaussian function fitting approach: profile (a), contact of three phases (b), base (c). 

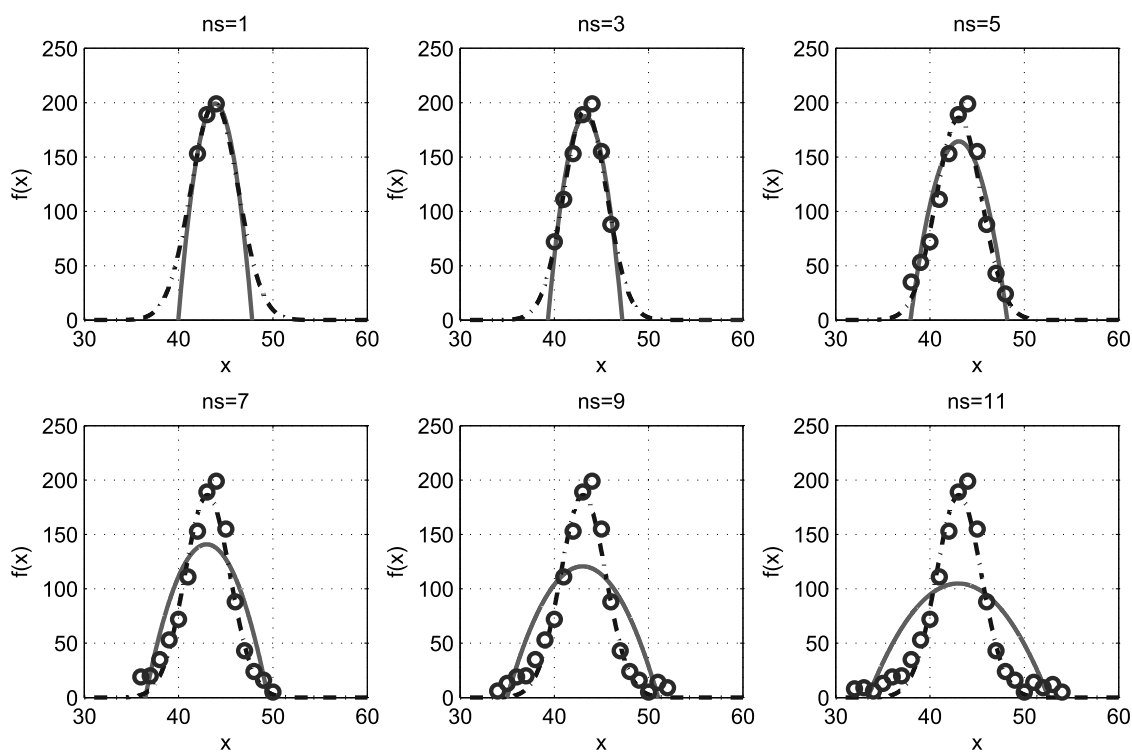

Fig. 20. Influence of the neighborhood size on the shape of the reconstructing function.

rounded to the closest pixel.

The comparison of the resulting edges with the original images again proves superiority of the Gaussian function fitting method over the gravity center and parabola fitting approaches. Based on a visual assessment, it can be concluded that the Gaussian function fitting approach produces an edge which describes the specimen profile most accurately. This can be observed especially in the contact of three phases where the aura phenomenon is strengthened by an overlap effect in the horizontal and vertical directions. In this region, the parabola fitting approach is unstable and produces an irregular edge. The profile provided by the gravity center approach visibly moves away from the border of a specimen. Only the Gaussian fitting approach yields reasonable results as it matches specimen shape and is most resistant to an aura.

6.2. Determination of surface tension. Results of surface tension determination from images of copper and silver using pixel and subpixel processing are shown in Figs. 22 and 23, and Table 3. Specifically, the determined values of surface tension of copper in the function of temperature (1400 K-1600 K) are shown in Fig. 22(a) while the determined values of surface tension of silver in function of temperature $(1260 \mathrm{~K}-1440 \mathrm{~K})$ are shown in Fig. 23(a). The corresponding trade lines are presented in Figs. 22(b) and 23(b), respectively. The parameters of trend equations in the form $\gamma=p+q T[\mathrm{~K}]$ (where $T$ denotes temperature) are indicated in Table 3 . Results provided by the gravity center approach (series $g c$, lightgray line) and the proposed Gaussian fitting approach (series gauss, mid-gray line) are compared with those obtained from the coarse edge (series coarse, dark-gray line)
Table 3. Trend lines equations for changes of surface tension in the function of temperature.

\begin{tabular}{|c||c|c|c|c|}
\hline \multicolumn{1}{|c||}{ Method } & \multicolumn{2}{c|}{ Copper } & \multicolumn{2}{c|}{ Silver } \\
\hline & $p$ & $q$ & $p$ & $q$ \\
\hline \hline reference & -0.2280 & 1207.1 & -0.2300 & 1609.3 \\
\hline coarse & -0.3661 & 1418.0 & -0.1644 & 1536.7 \\
\hline gc & 0.0255 & 940.5 & -0.1415 & 1596.6 \\
\hline gauss & -0.2295 & 1217.8 & -0.1808 & 1542.7 \\
\hline
\end{tabular}

and the referential values of surface tension (Mills and $\mathrm{Su}, 2006)$ (series reference, black line). Due to high instability and low accuracy of gradient profile reconstruction, the parabola fitting approach was excluded from the comparison.

Numerical results presented in Figs. 22 and 23 as well as Table 3 confirm the conclusions from the visual assessment (see Fig. 21) that the Gaussian fitting approach outperforms the other methods for edge detection. Specifically, the specimen shape description at the subpixel level using the proposed method increased the accuracy of surface tension measurements.

The gravity center approach failed in both cases considered. In the case of copper, it produced results of a character compliant with the laws of physics (i.e., surface tension decreases with temperature (Adamson and Gast, 1997)). However, the determined values of surface tension are significantly higher than the referential ones. In the case of silver, the trend of results is improper as surface tension increases with temperature.

The determined values of surface tension obtained using the proposed method in both cases visibly decrease with temperature, which is consistent with the general 

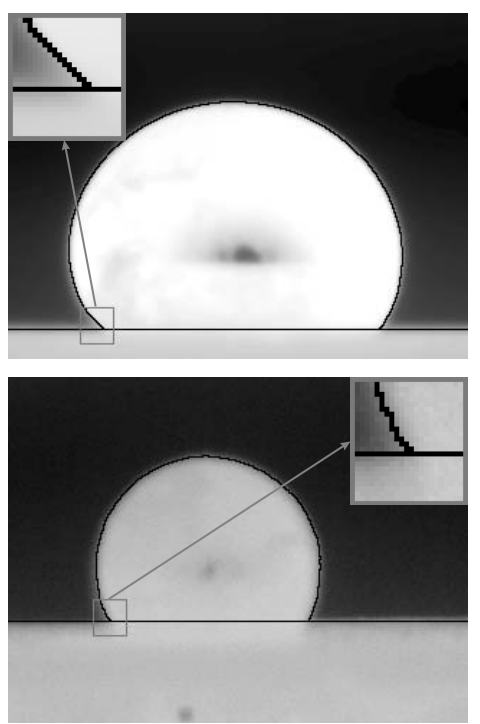

(a)
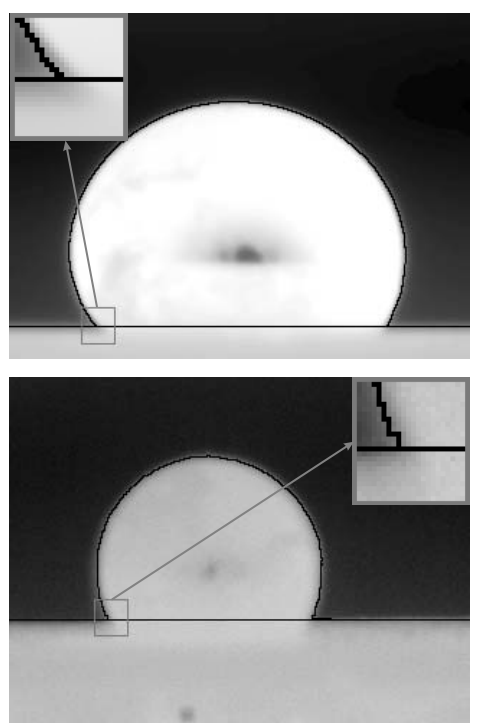

(b)
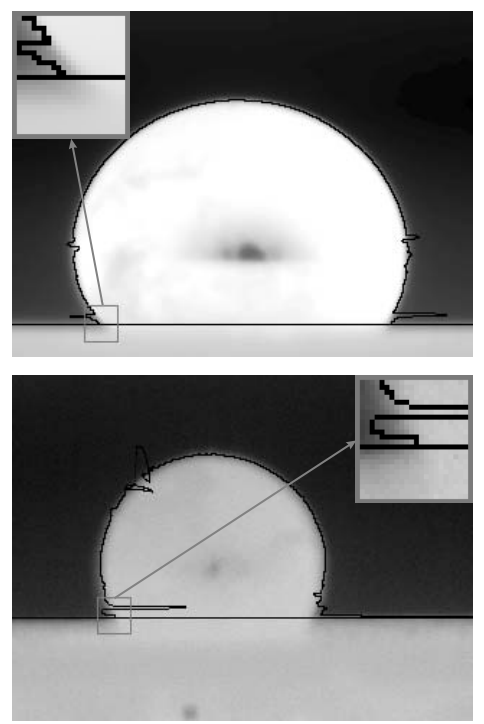

(c)

Fig. 21. Results of edge detection in the images from Fig. 5: top panel—silver (Fig. 5(b)), bottom panel—copper (Fig. 5(a)). Gravity center approach (a), Gaussian function fitting approach (b), parabola fitting approach (c).

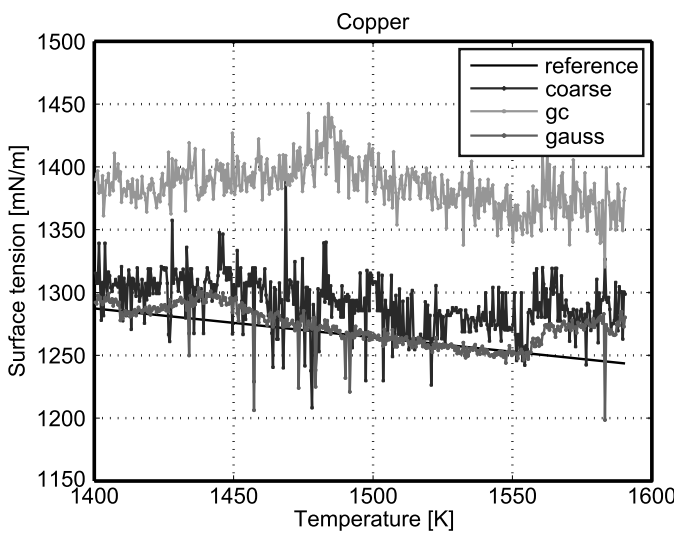

(a)

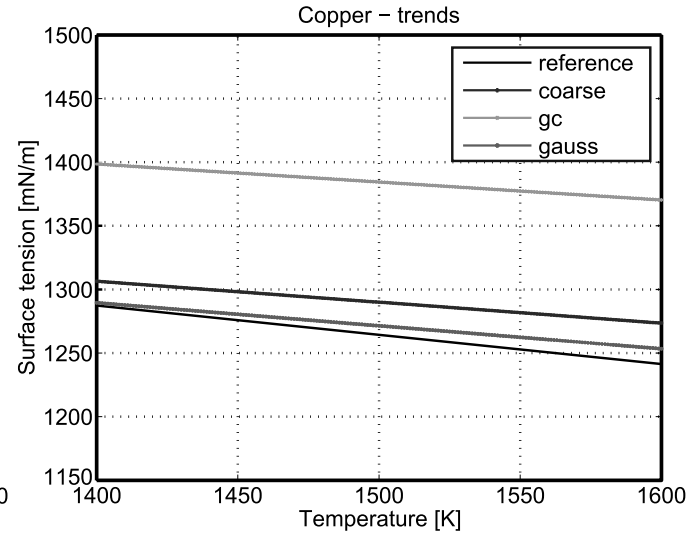

(b)

Fig. 22. Results of surface tension determination from images of copper: determined values of surface tension (a), trend lines (b).

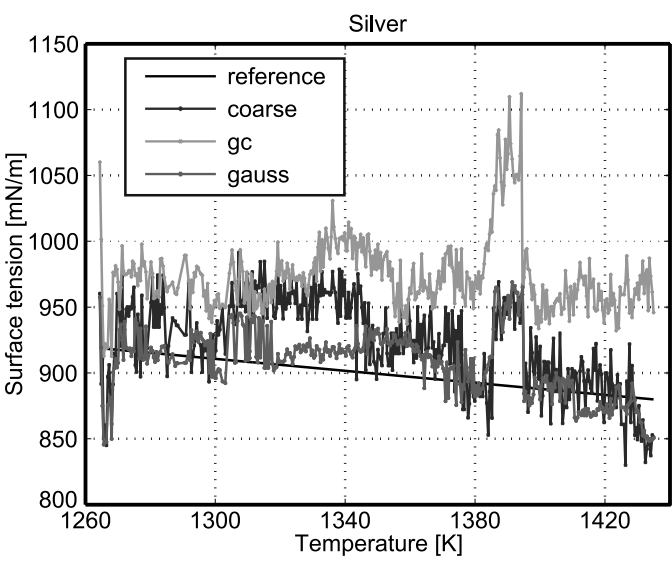

(a)

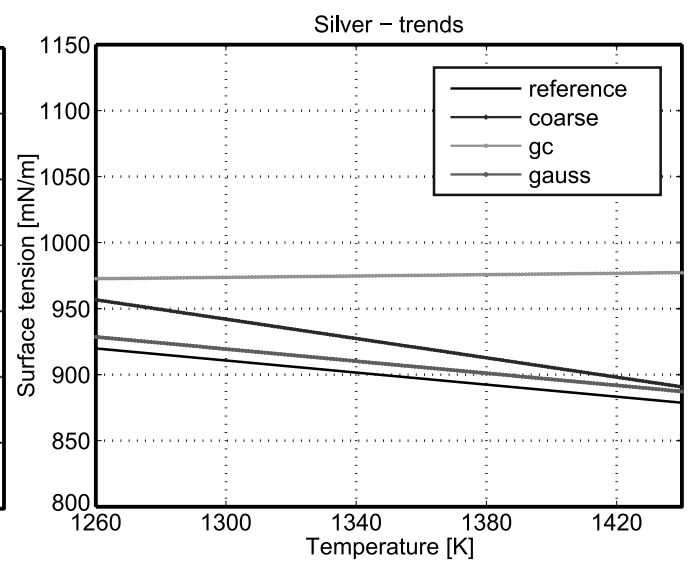

(b)

Fig. 23. Results of surface tension determination from images of silver: determined values of surface tension (a), trend lines (b). 
trend known from surface tension theory. Additionally, trend lines obtained using the Gaussian function fitting approach are remarkably closer to the referential trend line than the series obtained using image processing at the pixel level. This qualitative assessment is confirmed by equations of trend lines (see Table 3 ).

At the end it should be underlined that there is no "gold standard" for surface tension of metals. The hightemperature measurements of surface tension are related to different problems arising from the activity of liquid metals and sensitivity of surface phenomena to impurities and the measurement conditions (temperature, pressure, atmosphere, etc.). Therefore, recent results vary slightly depending on the measurement method (Mills and $\mathrm{Su}, 2006)$. However, despite this uncertainty, the presented comparison proves the superiority of the proposed subpixel processing in case of the measurement system considered.

\section{Conclusions}

In this paper the problem of edge detection with subpixel accuracy in images of heat-emitting specimens of metals was considered. The algorithm for refining edges to the subpixel level was proposed. The method uses a Gaussian function to reconstruct image information in a neighborhood of the coarse edge and to determine the subpixel position of edge points. Next, subpixel edge points are linked by cubic splines in order to obtain a continuous border.

The proposed method was successfully verified on a target measurement system. Specifically, applying the proposed method in a computer vision system for high temperature measurements of surface properties of metals and alloys increased the accuracy of surface tension determination. Additionally, the results provided by the Gaussian fitting approach were remarkably better than those provided by previously proposed approaches to subpixel edge detection.

Although the proposed method of edge detection with subpixel accuracy was developed for a certain industrial measurement system, it can be easily adapted to a wide range of computer vision systems where accurate edge detection is at premium. Additionally, it can be particularly useful in applications where the exact position of a blurred and unsharp edge must be precisely determined.

\section{Acknowledgment}

The presented work is supported by the Ministry of Science and Higher Education of Poland through founds for science for the years 2010-2012 as a research project no. N N516 490439. Additionally, the author receives financial support from the Foundation for Polish Science in the framework of the START fellowship.

\section{References}

Adamson, A. and Gast, A. (1997). Physical Chemistry of Surface, Wiley, Hoboken, NJ.

Bachevsky, R. S., Naidich, Y. V., Grygorenko, M. F. and Dostojny, V. A. (1994). Evaluation of errors in automatic image analysis determination of sessile drop shapes, Proceedings of the International Conference on High Temperature Capillarity, Sanremo, Italy, pp. 254-258.

Bailey, D.G. (2005). Sub-pixel profiling, Proceedings of the 5th International Conference on Information Communications and Signal Processing, Bangkok, Thailand, pp. 13111315.

Batchelor, B. and Whelan, P. (2002). Intelligent Vision Systems for Industry, http: / / elm. eeng. dcu . ie/

$\sim$ whelanp/ivsi/.

Bie, H.X. and Liu C.Y. (2009). Edge-directed sub-pixel and still image super-resolution, Proceedings of the 2nd International Congress on Image and Signal Processing, Tianjin, China, pp. 1-4

Bin, T.J., Lei, A., Jiwen, C., Wenjing, K. and Dandan, L. (2008). Subpixel edge location based on orthogonal Fourier-Mellin moments, Image and Vision Computing 26(4): $563-569$.

Breder R., Estrela V.-V. and de Assis J.T. (2009). Sub-pixel accuracy edge fitting by means of b-spline, Proceedings of the IEEE International Workshop on Multimedia Signal Processing, Rio De Janeiro, Brazil, pp. 1-5.

Dennis, J.E. andSchnabel, R.B. (1983). Numerical Methods for Unconstrained Optimization and Nonlinear Equations, Prentice Hall, Upper Saddle River, NJ.

Extrand, C.W. and Moon, S.I. (2010). When sessile drops are no longer small: Transitions from spherical to fully flattened, Langmuir 26(14): 11815-11822.

Fabijańska, A. and Sankowski, D. (2009). Computer vision system for high temperature measurements of surface properties, Machine Vision and Applications 20(6): 411-421.

Fabijańska, A. and Sankowski, D. (2010). Edge detection with sub-pixel accuracy in images of molten metals, IEEE International Conference on Imaging Systems and Techniques, Thessaloniki, Greece, pp. 186-191.

Gocławski, J., Sekulska-Nalewajko, J., Gajewska, E. and Wielanek, M. (2009). An automatic segmentation method for scanned images of wheat root systems with dark discolourations, International Journal of Applied Mathematics and Computer Science 19(4): 679-689, DOI: 10.2478/v10006-009-0055-x.

Gonzalez, R.C. and Woods, R.E. (2007). Digital Image Processing, 3rd Edn., Prentice Hall, Upper Saddle River, NJ.

Ghosal, S. and Mehrotra, R. (1993). Orthogonal moment operators for subpixel edge detection, Pattern Recognition Letters 26(2): 295-305.

Hansen, F.K. (1993). Surface tension by image analysis: Fast and automatic measurements of pendant and sessile drops and bubbles, Journal of Colloid and Interface Science 160(1): 209-217. 
Huh, C. and Reed, R.L. (1983). A method for estimating interfacial tensions and contact angles from sessile and pendant drop shapes, Journal of Colloid and Interface Science 91(2): 472-484.

Ji X., Wang, K. and Wei, Z. (2009). Structured light encoding research based on sub-pixel edge detection, Proceedings of the International Conference on Information Engineering and Computer Science, Wuhan, China, pp. 1-4.

Jin, J.S. (1990). An adaptive algorithm for edge detection with subpixel accuracy in noisy images, Proceedings of the IAPR Workshop on Machine Vision Applications, Tokyo, Japan, pp. 249-252.

Kisworo, M., Venkatesh, S. and West, G. (1991). 2-D edge feature extraction to subpixel accuracy using the generalized energy approach, Proceedings of the IEEE Region 10 International Conference on EC3-Energy, Computer, Communication and Control Systems, New Delhi, India, pp. 344348.

Koszmider, T., Strzecha, K., Fabijańska, A. and Bakala, M. (2011). Algorithm for accurate determination of contact angles in vision system for high temperature measurements of metals and alloys surface properties, in R. Burduk, M. Kurzyński, M. Woźniak and A. Żołnierek (Eds.), Computer Recognition Systems 4, Advances in Intelligent and Soft Computing, Vol. 95, Springer, Berlin/Heidelberg, pp. 441-448, DOI: 10.1007/978-3-642-20320-6_46.

Liu, C., Xia Z., Niyokindi, S., Pei, W., Song, J. and Wang, L. (2004). Edge location to sub-pixel value in color microscopic images, Proceedings of the International Conference on Intelligent Mechatronics and Automation, Chengdu, Sichuan, China, pp. 548-551.

Lyvers, E.P., Mitchell, O.R., Akey, M.L. and Reeves, A.P. (1989). Subpixel mesurements using a moment-basededge operator, IEEE Transactions on Pattern Analysis and Machine Intelligence 11(12): 1293-1309.

Machuca, R. and Gilbert, A.L. (1981). Finding edges in noisy scenes, IEEE Transactions on Pattern Analysis and Machine Intelligence 3(1): 103-111.

MacVicar-Whelan, P.J. and Binford, T.O. (1981). Intensity discontinuity location to subpixel precision, Proceedings of the International Joint Conference on Artificial Intelligence, Vancouver, BC, Canada, pp. 26-31.

MacVicar-Whelan, P.J. and Binford, T.O. (1991). Line finding with subpixel precision, Proceedings of the DARPA Image Understanding Workshop, Washington, DC, USA, pp. 2631.

Mills, K.C. and Su, Y.C (2006). Review of surface tension data for metallic elements and alloys, Part 1: Pure metals, International Materials Reviews 51(6): 329-351.

Nevtia, R. and Babu, K. (1978). Linear feature extraction, Proceedings of the DARPA Image Understanding Workshop, Pittsburgh, PA, USA, pp. 73-78.

Obinata, G. and Dutta, A. (2007). Vision Systems: Applications, I-Tech Education and Publishing, Vienna.

Oskoei, M.A. and Hu, H. (2010). A survey on edge detection methods, Technical Report CES-506, University of Essex, Essex.
Ranky, P. (2003). Advanced machine vision systems and application examples, Sensor Review 23(3): 242-245.

Ridler, T. and Calvard, S. (1978). Picture thresholding using an iterative selection method, IEEE Transations on Systems, Man, and Cybernetics 8(8): 630-632

Rocket, P. (1999). The accuracy of sub-pixel localization in the Canny edge detector, Proceedings of the British Machine Vision Conference, Nottingham, UK, pp. 392-401.

Sankowski, D., Strzecha, K. and Jezewski, S. (2000). Digital image analysis in measurement of surface tension and wettability angle, Proceedings of the International Conference on Modern Problems of Telecommunications, Computer Science and Engineers Training, Lviv/Slavsko, Ukraine, pp. 129-130.

Scott, N. (2010). Remote Sensing Tutorial, http://rst.gsfc.nasa.gov/Sect13/ Sect13_2.html.

Senthilkumaran, N. and Rajesh, R. (2009). Edge detection techniques for image segmentation: A survey of soft computing approaches, International Journal of Recent Trends in Engineering 1(2): 250-254.

Sheng, Y. and Shen, L. (1994). Orthogonal Fourier-Mellin moments for invariant pattern recognition, Journal of the Optical Society of America 11(6): 1748-1757.

Sidiropoulos, N.D., Baras, J.S. and Berenstein, C.A. (1992). Discrete random sets: An inverse problem, plus tools for the statistical inference of the discrete Boolean model, Proceedings of SPIE 1769(1):32-43.

Stanke, G., Zedler, L., Zorn, A., Weckend, F. and Weide, H.G. (1998). Sub-pixel accuracy by optical measurement of large automobile components, Proceedings of the 24th Annual Conference of the IEEE Industrial Electronics Society, Aachen, Germany, pp. 2431-2433.

Steger, C., Ulrich, M. and Wiedemann, C. (2008). Machine Vision Algorithms and Applications, Wiley-VCH, Berlin.

Strzecha, K., Bakala, M., Fabijańska, A. and Koszmider. T (2010). The evolution of Thermo-Wet: The computerized system for measurements of surface properties, $\mathrm{Au}$ tomatyka 3(14): 525-535, (in Polish).

Tabatabai, A.J. and Mitchell, O.R. (1984). Edge location to subpixel values in digital imagery, IEEE Transactions on Pattern Analysis and Machine Intelligence 6(2): 188-201.

$\mathrm{Xu}$, G.S. (2009a). Sub-pixel edge detection based on curve fitting, Proceedings of the 2nd International Conference on Information and Computing Science, Manchester, UK, pp. 373-375.

Xu, G.S. (2009b). Linear array CCD image sub-pixel edge detection based on wavelet transform, Proceedings of the 2 nd International Conference on Information and Computing Science, Manchester, UK, pp. 204-206.

Yao, Y. and Ju, H. (2009). A sub-pixel edge detection method based on canny operator, Proceedings of the 6th International Conference on Fuzzy Systems and Knowledge Discovery, Tianjin, China, pp. 97-100. 


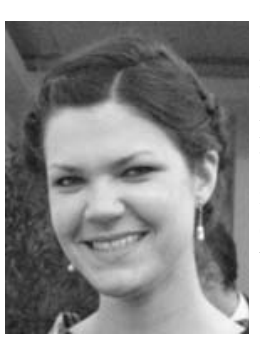

Anna Fabijańska is an assistant professor at the Institute of Applied Computer Science, Łódź University of Technology (Poland). She received her Ph.D. in computer science from the Łódź University of Technology in 2007. Her research interests focus on the development of image processing and analysis algorithms for industrial and biomedical vision systems.

Received: 7 July 2011

Revised: 16 December 2011 